PROCEEDINGS OF THE

AMERICAN MATHEMATICAL SOCIETY

Volume 135, Number 3, March 2007, Pages 851-860

S 0002-9939(06)08530-3

Article electronically published on September 11, 2006

\title{
NON-INTERSECTION BODIES, ALL OF WHOSE CENTRAL SECTIONS ARE INTERSECTION BODIES
}

\author{
M. YASKINA \\ (Communicated by N. Tomczak-Jaegermann)
}

\begin{abstract}
We construct symmetric convex bodies that are not intersection bodies, but all of their central hyperplane sections are intersection bodies. This result extends the studies by Weil in the case of zonoids and by Neyman in the case of subspaces of $L_{p}$.
\end{abstract}

\section{INTRODUCTION}

The concept of an intersection body was introduced by Lutwak $\mathrm{Lu}$ in 1988. Let $K$ and $L$ be origin symmetric star bodies in $\mathbb{R}^{n}$. Following $[\mathrm{Lu}$, we say that $K$ is the intersection body of $L$ if the radius of $K$ in every direction is equal to the volume of the central hyperplane section of $L$ perpendicular to this direction, i.e. for every $\xi \in S^{n-1}$,

$$
\|\xi\|_{K}^{-1}=\operatorname{vol}_{n-1}\left(L \cap \xi^{\perp}\right) .
$$

The closure in the radial metric of the class of intersection bodies of star bodies gives the class of intersection bodies.

Intersection bodies played an important role in the solution of the BusemannPetty problem (see [K5, Chapter 5] for the solution and [K5, Chapter 1] for the historical details). Posed in 1956, $\mathrm{BP}$, the Busemann-Petty problem asks the following. Let $K$ and $L$ be two origin-symmetric convex bodies in $\mathbb{R}^{n}$ so that the $(n-1)$-dimensional volume of every central hyperplane section of $K$ is smaller than the same for $L$. Does it follow that the $n$-dimensional volume of $K$ is smaller than the $n$-dimensional volume of $L$ ? The answer turns out to be affirmative for dimensions $n \leq 4$ and negative for $n \geq 5$.

The connection between intersection bodies and the Busemann-Petty problem was found by Lutwak $\mathrm{Lu}$. First, the answer to the problem is affirmative if $K$ is an intersection body and $L$ is any origin-symmetric star body. On the other hand, if $L$ is an origin-symmetric convex body that is not an intersection body, one can perturb $L$ to construct a counterexample to the Busemann-Petty problem. Hence, a solution to the problem in $\mathbb{R}^{n}$ is affirmative if and only if every infinitely smooth origin-symmetric convex body in $\mathbb{R}^{n}$ is an intersection body, which is the case for dimensions $n \leq 4$. Examples of non-intersection bodies in dimensions 5 and higher were constructed in [Ga1], Zh1], GKS, [K3].

Received by the editors May 12, 2005 and, in revised form, October 3, 2005.

2000 Mathematics Subject Classification. Primary 52A20, 52A21, 46B20.

(C)2006 American Mathematical Society 
In this paper we are interested in the following problem. Does there exist a convex body $K$ that is not an intersection body, but its section by a central hyperplane is an intersection body? We construct an example of such a body for dimensions $n \geq 5$. Our result can also be considered as a new way of constructing non-intersection bodies.

This paper was motivated by results of W.Weil [W] and A.Neyman [N]. In 1982 W.Weil, [W], showed that it is not possible to characterize zonoids by means of their projections. He constructed a convex body in $\mathbb{R}^{n}(n \geq 3)$ that is not a zonoid but all its projections onto hyperplanes are zonoids. A.Neyman in [N] showed that there are $n$-dimensional normed spaces that do not embed in $L_{p}$, but all their $(n-1)$-dimensional subspaces embed in $L_{p}$ for $p>0$. He used this to prove that for $p>0, p \neq 2, L_{p}$ is not characterized by a finite number of equations. Let us note that M.Burger in [B] used another approach to show that, for $n \geq 3$, zonoids cannot be characterized by a finite number of piecewise inequalities. A.Koldobsky in [K4] introduced the concept of embedding of normed spaces in $L_{p}, p<0$, and proved that intersection bodies are the unit balls of spaces that embed in $L_{-1}$. Therefore, our result can be considered as an extension of Neyman's example to negative $p$.

\section{MAIN RESULTS}

Our main tool is the Fourier transform of distributions. The Fourier transform of a distribution $f$ is defined by $\langle\hat{f}, \phi\rangle=\langle f, \hat{\phi}\rangle$ for every test function $\phi$ from the Schwartz space $\mathcal{S}$ of rapidly decreasing infinitely differentiable functions on $\mathbb{R}^{n}$. For any even distribution $f$, we have $(\hat{f})^{\wedge}=(2 \pi)^{n} f$.

A distribution is positive definite if its Fourier transform is a positive distribution in the sense that $\langle\hat{f}, \phi\rangle \geq 0$ for every non-negative test function $\phi$; see, for example, GV, p. 152].

Let $K$ be a convex origin-symmetric body in $\mathbb{R}^{n}$. Our definition of a convex origin-symmetric body assumes that the origin is an interior point of $K$. The radial function of $K$ is given by

$$
\rho_{K}(x)=\max \{a>0: a x \in K\}, \quad x \in \mathbb{R}^{n} \backslash\{0\} .
$$

The Minkowski norm of $K$ is defined by

$$
\|x\|_{K}=\min \{a \geq 0: x \in a K\} .
$$

Clearly $\rho_{K}(x)=\|x\|_{K}^{-1}$.

The main result of this paper is the following.

Theorem 2.1. There exists a convex body $K$ in $\mathbb{R}^{n}, n \geq 5$, that is not an intersection body, but for every $(n-1)$-dimensional subspace $V$ of $\mathbb{R}^{n}, K \cap V$ is an intersection body.

To construct an example of such a body we use a connection between the Fourier transform and intersection bodies. A.Koldobsky in $\mathrm{K} 2$ proved that an originsymmetric star body $K$ in $\mathbb{R}^{n}$ is an intersection body if and only if $\|x\|_{K}^{-1}$ is a positive definite distribution.

We will use Lemma 3.16 from [K5, p. 58]. It states the following. 
Lemma 2.2. Let $k \in \mathbb{N} \cup\{0\}$ and $f \in C^{2 k}\left(S^{n-1}\right)$, where $f$ is even, $q \leq 2 k$, and $q$ is not an odd integer. Let $x=(r, \theta)$ be polar coordinates in $\mathbb{R}^{n}$, so that $f_{\varepsilon}(\theta) r^{p}=f_{\varepsilon}\left(\frac{x}{|x|_{2}}\right)|x|_{2}^{-p}$. Then:

(i) The Fourier transform of the distribution $f(\theta) r^{-n+q+1}$ is a homogeneous of degree $-1-q$ continuous on $\mathbb{R}^{n} \backslash\{0\}$ function. If $q<2 k$, then for every $x \in \mathbb{R}^{n}$,

$$
\begin{aligned}
|x|_{2}^{2 k}( & \left.f(\theta) r^{-n+q+1}\right)^{\wedge}(x)=\frac{(-1)^{k} \pi}{-2 \Gamma(2 k-q) \sin (\pi(2 k-q-1) / 2)} \\
& \left.\times \int_{S^{n-1}}|(x, \xi)|^{2 k-q-1} \Delta^{k}\left(f(\theta) r^{-n+q+1}\right)\right)(\xi) d \xi .
\end{aligned}
$$

If $q=2 k$, then

$$
\begin{aligned}
& \left(f(\theta) r^{-n+q+1}\right)^{\wedge}(x)=(-1)^{k} \pi|x|_{2}^{-1-2 k} \\
\times & \int_{S^{n-1} \cap\left(x /|x|_{2}\right)^{\perp}} \Delta^{k}\left(f(\theta) r^{-n+q+1}\right)(\xi) d \xi,
\end{aligned}
$$

where $\Delta$ is the Laplace operator in $\mathbb{R}^{n}$.

(ii) If $f \in C^{\infty}\left(S^{n-1}\right)$, then there exist an even function $g \in C^{\infty}\left(S^{n-1}\right)$ so that for every $x=t \xi \in \mathbb{R}^{n}, t \neq 0, \xi \in S^{n-1}$,

$$
\left(f(\theta) r^{-n+q+1}\right)^{\wedge}(x)=g(\xi) t^{-1-q},
$$

so the Fourier transform of $f(\theta) r^{-n+q+1}$ is an infinitely smooth function on $\mathbb{R}^{n} \backslash\{0\}$.

Fix a point $x_{0}$ on the unit sphere $S^{n-1}$. Define a function $f_{\varepsilon}$ as follows:

$$
f_{\varepsilon}(x)= \begin{cases}2 e^{-\frac{\left|x-x_{0}\right|^{2}}{\varepsilon^{2}-\left|x-x_{0}\right|^{2}}} & \text { if }\left|x-x_{0}\right|<\varepsilon, \\ 2 e^{-\frac{\left|x+x_{0}\right|^{2}}{\varepsilon^{2}-\left|x+x_{0}\right|^{2}}} & \text { if }\left|x+x_{0}\right|<\varepsilon \\ 0 & \text { otherwise. }\end{cases}
$$

Clearly $f_{\varepsilon}$ is an infinitely differentiable function. Define a body $K$ by

$$
\|x\|_{K}^{-1}=\left(\left(1-f_{\varepsilon}(\theta)\right) r^{-n+1}\right)^{\wedge}(x), \quad x \in \mathbb{R}^{n} \backslash\{0\} .
$$

The function $\|x\|_{K}^{-1}$ is infinitely smooth on $\mathbb{R}^{n} \backslash\{0\}$ by Lemma 2.2, It will be shown in Lemma 2.4 that $\left(f_{\varepsilon}(\theta) r^{-n+1}\right)^{\wedge}(x)$ is of the order $\varepsilon^{n-2}$ uniformly with respect to $x \in S^{n-1}$ and the Fourier transform of $r^{-n+1}$ is strictly greater then zero, therefore $\|x\|_{K}$ is positive for a small $\varepsilon$.

Lemma 2.3. For any $\varepsilon>0, K$ is not an intersection body.

Proof. Since $f_{\varepsilon}$ is an even function, we have

$$
\left(\|x\|_{K}^{-1}\right)^{\wedge}=(2 \pi)^{n}\left(1-f_{\varepsilon}(\theta)\right) r^{-n+1},
$$

which is negative for $\theta$ in some neighborhood of $x_{0}$. Therefore, by [K2, Theorem $1], K$ is not an intersection body. 
Lemma 2.4. There exist numbers $D_{1}, D_{2}, D_{3}$ that do not depend on $\varepsilon$ so that for every $x \in S^{n-1}$

$$
\begin{gathered}
\left|\left(f_{\varepsilon}(\theta) r^{-n+1}\right)^{\wedge}(x)\right| \leq D_{1} \varepsilon^{n-2}, \\
\left|\frac{\partial}{\partial x_{i}}\left(f_{\varepsilon}(\theta) r^{-n+1}\right)^{\wedge}(x)\right| \leq D_{2}|\ln \varepsilon| \cdot \varepsilon^{n-3},
\end{gathered}
$$

and

$$
\left|\frac{\partial^{2}}{\partial x_{i} \partial x_{j}}\left(f_{\varepsilon}(\theta) r^{-n+1}\right)^{\wedge}(x)\right| \leq D_{3} \varepsilon^{n-4} .
$$

Proof. First we show the estimate for the second derivative. Using the connection between the Fourier transform and differentiation and Lemma 2.2 with $q=2$ and $k=1$, we get

$$
\begin{gathered}
\frac{\partial^{2}}{\partial x_{i} x_{j}}\left(f_{\varepsilon}(\theta) r^{-n+1}\right)^{\wedge}(x)=-\left(f_{\varepsilon}\left(\frac{y}{|y|}\right) y_{i} y_{j}|y|^{-n+1}\right)^{\wedge}(x) \\
=-\left(f_{\varepsilon}\left(\frac{y}{|y|}\right) \frac{y_{i} y_{j}}{|y|^{2}}|y|^{-n+3}\right)^{\wedge}(x)=-\left(g_{\varepsilon}(\theta)|y|^{-n+3}\right)^{\wedge}(x) \\
=\pi|x|_{2}^{-3} \int_{S^{n-1} \cap\left(x /|x|_{2}\right)^{\perp}} \triangle\left(g_{\varepsilon}(\theta)|y|^{-n+3}\right)(\xi) d \xi,
\end{gathered}
$$

where

$$
g_{\varepsilon}(\theta)=f_{\varepsilon}\left(\frac{y}{|y|}\right) \frac{y_{i} y_{j}}{|y|^{2}}, \quad \theta=\frac{y}{|y|} \in S^{n-1} .
$$

Note that the function $g_{\varepsilon}(\theta)$ is supported in $B_{\epsilon}\left(x_{0}\right)$ and $B_{\epsilon}\left(-x_{0}\right)$, where

$$
B_{\epsilon}\left(x_{0}\right)=\left\{x \in S^{n-1}:\left|x-x_{0}\right|<\varepsilon\right\} .
$$

The volume of these balls is of the order $\varepsilon^{n-1}$.

Now we want to show that $\frac{\partial^{2}}{\partial x_{i} \partial x_{j}}\left(f_{\varepsilon}(\theta) r^{-n+1}\right)^{\wedge}(x)$ can be made as small as desirable if $\varepsilon$ is small. First let us show that $\frac{\partial^{2}}{\partial y_{i}^{2}}\left(g_{\varepsilon}\left(\frac{y}{|y|}\right)\right)$ is of the order $\varepsilon^{-2}$.

To prove this, consider the function

$$
f_{1}(x)= \begin{cases}2 e^{-\frac{\left|x-x_{0}\right|^{2}}{1-\left|x-x_{0}\right|^{2}}} & \text { if }\left|x-x_{0}\right|<1 \\ 2 e^{-\frac{\left|x+x_{0}\right|^{2}}{1-\left|x+x_{0}\right|^{2}}} & \text { if }\left|x+x_{0}\right|<1 \\ 0 & \text { otherwise }\end{cases}
$$

where $x \in S^{n-1}$.

The function $f_{1}$ is infinitely differentiable, so all its derivatives are bounded. Also $f_{1}\left(\frac{x /|x|-x_{0}}{\varepsilon}+x_{0}\right)=f_{\varepsilon}(x /|x|)$. Therefore,

$$
\left|\frac{\partial^{k}}{\partial x_{i}^{k}} f_{\varepsilon}(x /|x|)\right|=\left|\frac{\partial^{k}}{\partial x_{i}^{k}} f_{1}\left(\frac{x /|x|-x_{0}}{\varepsilon}+x_{0}\right)\right| \leq C_{k} \varepsilon^{-k},
$$

where $C_{k}$ depends on $k$ but not on $x$.

The same is true for the derivatives of $g_{\varepsilon}(\theta)=f_{\varepsilon}\left(\frac{y}{|y|}\right) \frac{y_{i}^{2}}{|y|^{2}}$, i.e.

$$
\left|\frac{\partial^{k}}{\partial y_{i}^{k}} g_{\varepsilon}\left(\frac{y}{|y|}\right)\right| \leq \tilde{C}_{k} \varepsilon^{-k} .
$$


So $\triangle\left(g_{\varepsilon}(\theta)|y|^{-n+3}\right)(\xi)$ is also of the order $\varepsilon^{-2}$ since the main term when $\varepsilon$ is small is $\frac{\partial^{2}}{\partial y_{i}^{2}}\left(g_{\varepsilon}\left(\frac{y}{|y|}\right)\right)|y|^{-n+3}$.

Therefore,

$$
\begin{gathered}
\left|\int_{S^{n-1} \cap\left(x /|x|_{2}\right)^{\perp}} \triangle\left(g_{\varepsilon}(\theta)|y|^{-n+3}\right)(\xi) d \xi\right| \\
\leq \tilde{C}_{2} \sup \left|\triangle\left(g_{\varepsilon}(\theta)|y|^{-n+3}\right)\right| \cdot \int_{\left(B_{\varepsilon}\left(x_{0}\right) \cup B_{\varepsilon}\left(-x_{0}\right)\right) \cap\left(x /|x|_{2}\right)^{\perp}} d \\
=O\left(\varepsilon^{-2} \varepsilon^{n-2}\right)=O\left(\varepsilon^{n-4}\right),
\end{gathered}
$$

since the volume of the balls $B_{\varepsilon}\left(x_{0}\right)$ and $B_{\varepsilon}\left(-x_{0}\right)$ is of the order $\varepsilon^{n-1}$ and the volume of their intersection with a hyperplane is of the order $\varepsilon^{n-2}$.

So, using equality (2) we get $\frac{\partial^{2}}{\partial x_{i} \partial x_{j}}\left(f_{\varepsilon}(\theta) r^{-n+1}\right)^{\wedge}(x)=O\left(\varepsilon^{n-4}\right)$, and for $n>4$ it can be made as small as desirable uniformly with respect to $x \in S^{n-1}$.

Using the same argument, we prove that $\left(f_{\varepsilon}(\theta) r^{-n+1}\right)^{\wedge}(x)=O\left(\varepsilon^{n-2}\right)$.

To get the estimate for $\frac{\partial}{\partial x_{i}}\left(f_{\varepsilon}(\theta) r^{-n+1}\right)^{\wedge}(x)$ we again use Lemma 2.2 and the connection between the Fourier transform and differentiation. Take $\alpha$ close to 1 . Then, if $g_{\varepsilon}(\theta)=f_{\varepsilon}\left(\frac{y}{|y|}\right) \frac{y_{i}}{|y|}, \quad \theta=\frac{y}{|y|} \in S^{n-1}$,

$$
\begin{aligned}
& \frac{\partial}{\partial x_{i}}\left(f_{\varepsilon}(\theta) r^{-n+\alpha+1}\right)^{\wedge}(x)=-\left(g_{\varepsilon}(\theta)|y|^{-n+\alpha+2}\right)^{\wedge}(x) \\
& =\frac{-\pi|x|_{2}^{-2} \int_{S^{n-1}}|(x, \xi)|^{1-\alpha} \Delta\left(g_{\varepsilon}(\theta)|y|^{-n+\alpha+1}\right)(\xi) d \xi}{2 \Gamma(2-\alpha) \sin \frac{\pi(1-\alpha)}{2}} .
\end{aligned}
$$

When $\alpha$ approaches 1 , the numerator and denominator on the right-hand side approach zero. Indeed, let us show that the limit of the numerator is zero:

$$
\lim _{\alpha \rightarrow 1} \int_{S^{n-1}}|(x, \xi)|^{1-\alpha} \Delta\left(g_{\varepsilon}(\theta)|y|^{-n+\alpha+1}\right)(\xi) d \xi=\int_{S^{n-1}} \Delta\left(g_{\varepsilon}(\theta)|y|^{-n+2}\right)(\xi) d \xi .
$$

Recall the relation between the spherical Laplacian $\Delta_{S}$ and Euclidean Laplacian $\Delta$ (see, for example, $[\mathrm{Gr}$, p. 7]): if $f$ is a homogeneous function of degree $m$, then on the sphere

$$
\Delta_{S} f_{1}=\Delta f_{1}-m(m+n-2) f_{1} .
$$

Since $g_{\varepsilon}(\theta)|y|^{-n+2}$ has degree of homogeneity $-n+2$, the previous formula implies $\Delta\left(g_{\varepsilon}(\theta)|y|^{-n+2}\right)(\xi)=\Delta_{S}\left(g_{\varepsilon}(\theta)|y|^{-n+2}\right)(\xi)$. Due to the fact that $\Delta_{S}$ is a self-adjoint operator, Gr, p. 7], we have

$$
\int_{S^{n-1}} \Delta_{S}\left(g_{\varepsilon}(\theta)|y|^{-n+2}\right)(\xi) d \xi=0 .
$$

Now to compute the limit of (므) as $\alpha \rightarrow 0$, apply l'Hôpital's rule:

$$
\frac{\partial}{\partial x_{i}}\left(f_{\varepsilon}(\theta) r^{-n+2}\right)^{\wedge}(x)=|x|_{2}^{-2} \int_{S^{n-1}} \ln |(x, \xi)| \Delta\left(g_{\varepsilon}(\theta)|y|^{-n+2}\right)(\xi) d \xi .
$$

Recall that the function $g_{\varepsilon}(\theta)$ is supported in the balls $B_{\varepsilon}\left(x_{0}\right)$ and $B_{\varepsilon}\left(-x_{0}\right)$. Then

$$
\begin{aligned}
\left|\int_{S^{n-1}} \ln \right|(x, \xi) \mid \Delta & \left(g_{\varepsilon}(\theta)|y|^{-n+2}\right)(\xi) d \xi|\leq \sup | \Delta\left(g_{\varepsilon}(\theta)|y|^{-n+2}\right)(\xi) \mid \\
& \times \int_{B_{\varepsilon}\left(x_{0}\right) \cup B_{\varepsilon}\left(-x_{0}\right)}|\ln |(x, \xi)|| d \xi .
\end{aligned}
$$


Now we want to estimate the latter integral. Note that it is enough to estimate just $\int_{B_{\varepsilon}\left(x_{0}\right)}|\ln |(x, \xi) \| d \xi$. Consider two cases. First, suppose that $x$ is not perpendicular to any $y \in B_{2 \varepsilon}\left(x_{0}\right)$. In this case one can check that $|(x, \xi)|>\varepsilon / 2$ and therefore

$$
\int_{B_{\varepsilon}\left(x_{0}\right)}|\ln |(x, \xi)|| d \xi \leq|\ln (\varepsilon / 2)| \cdot \operatorname{vol}\left(B_{\varepsilon}\left(x_{0}\right)\right)=O\left(|\ln \varepsilon| \cdot \varepsilon^{n-1}\right),
$$

since the volume of the ball $B_{\varepsilon}\left(x_{0}\right)$ is of the order $\varepsilon^{n-1}$.

In the second case there exists $y \in B_{2 \varepsilon}\left(x_{0}\right)$ such that $x \perp y$. Consider the ball $B_{4 \varepsilon}(y)$. Clearly, $B_{2 \varepsilon}\left(x_{0}\right) \subset B_{4 \varepsilon}(y)$, therefore

$$
\int_{B_{\varepsilon}\left(x_{0}\right)}|\ln |(x, \xi)|| d \xi \leq \int_{B_{4 \varepsilon}(y)}|\ln |(x, \xi)|| d \xi .
$$

Let us make a change of coordinates from $\xi \in S^{n-1}$ to $\zeta \in S^{n-2}$ and $t \in$ $[-\pi / 2, \pi / 2]$ such that $\xi=y \sqrt{1-t^{2}}+\zeta t$. The Jacobian is equal to $\frac{t^{n-2}}{\sqrt{1-t^{2}}}$. If $\xi \in B_{4 \varepsilon}(y)$, then $t \in\left[0,4 \varepsilon \sqrt{1-4 \varepsilon^{2}}\right] \subset[0,4 \varepsilon]$. Using the fact that $x$ is perpendicular to $y$, we get

$$
\begin{aligned}
& \int_{B_{4 \varepsilon}(y)}|\ln |(x, \xi)|| d \xi \leq \int_{S^{n-2}} \int_{0}^{4 \epsilon}|\ln |(x, \zeta) t|| \frac{t^{n-2}}{\sqrt{1-t^{2}}} d t d \zeta \\
= & \int_{S^{n-2}}\left(\int_{0}^{4 \epsilon}|\ln |(x, \zeta)|| \frac{t^{n-2}}{\sqrt{1-t^{2}}} d t+\int_{0}^{4 \epsilon}|\ln t| \frac{t^{n-2}}{\sqrt{1-t^{2}}} d t\right) d \xi \\
& \leq 2 \int_{S^{n-2}}\left(\int_{0}^{4 \epsilon}|\ln |(x, \zeta)|| t^{n-2} d t+\int_{0}^{4 \epsilon}|\ln t| t^{n-2} d t\right) d \xi
\end{aligned}
$$

for a small $\varepsilon$. The first integral can be estimated in the following way:

$$
\int_{S^{n-2}}|\ln |(x, \zeta)|| d \xi \int_{0}^{4 \epsilon} t^{n-2} d t=O\left(\varepsilon^{n-1}\right) .
$$

Using integration by parts in the second integral, we get that

$$
\begin{aligned}
\int_{0}^{4 \epsilon}|\ln t| t^{n-2} d t= & \left.\frac{1}{n-1} \ln t \cdot t^{n-1}\right|_{0} ^{4 \varepsilon}-\frac{1}{n-1} \int_{0}^{4 \epsilon} t^{n-2} d t \\
& =O\left(|\ln \varepsilon| \cdot \varepsilon^{n-1}\right) .
\end{aligned}
$$

Therefore,

and

$$
\int_{B_{\varepsilon}\left(x_{0}\right) \cup B_{\varepsilon}\left(-x_{0}\right)}|\ln |(x, \xi)|| d \xi=O\left(|\ln \varepsilon| \cdot \varepsilon^{n-1}\right)
$$

$$
\frac{\partial}{\partial x_{i}}\left(f_{\varepsilon}(\theta) r^{-n+\alpha+1}\right)^{\wedge}(x)=O\left(|\ln \varepsilon| \cdot \varepsilon^{n-3}\right) .
$$

Lemma 2.5. If $n \geq 5$, the body $K$ is convex for small enough $\varepsilon$.

Proof. Geometrically the proof is based on the idea of adding in radial metric of an arbitrary small function to a radial function of the body which is uniformly convex, i.e. the curvature is a constant bounded from zero. In our case this body is the Euclidean ball. So, due to this property of a uniformly convex body, we can always choose a function in such a way that the body we get is still convex. 
Let $|\cdot|_{2}$ be the Euclidean norm. By [GS, p. 363] the Fourier transform of $|x|_{2}^{q}$, $q \in(-n, 0)$, equals

$$
\left(|x|_{2}^{q}\right)^{\wedge}(t)=2^{q+n} \pi^{n / 2} \frac{\Gamma\left(\frac{q+n}{2}\right)}{\Gamma\left(\frac{-q}{2}\right)}|t|_{2}^{-n-q} .
$$

Using this formula and the definition of the body $K$

$$
\begin{aligned}
\|x\|_{K}^{-1} & =\left(\left(1-f_{\varepsilon}(\theta)\right) r^{-n+1}\right)^{\wedge}(x) \\
& =C_{n}|x|_{2}^{-1}-\left(f_{\varepsilon}(\theta) r^{-n+1}\right)^{\wedge}(x),
\end{aligned}
$$

where $C_{n}=\frac{2 \pi^{(n+1) / 2}}{\Gamma\left(\frac{n-1}{2}\right)}$.

Let $K_{W}$ be the section of $K$ by a 2-dimensional central plane $W$ with an orthonormal basis $\xi_{1}, \xi_{2}$. So, if $x \in W \cap S^{n-1}$, then $x=\xi_{1} \cos \phi+\xi_{2} \sin \phi, \phi \in[0,2 \pi]$. To show that $K$ is convex, it is enough to show that $K_{W}$ is convex for any $W$.

Consider a function

$$
\rho_{W}(\phi)=\left(f_{\varepsilon}(\theta) r^{-n+1}\right)^{\wedge}\left(\xi_{1} \cos \phi+\xi_{2} \sin \phi\right) .
$$

By the definition of $K$, the radial function of $K_{W}$ is given by

$$
\rho(\phi)=C_{n}-\rho_{W}(\phi) .
$$

To prove that $K_{W}$ is convex, we need to show that for small $\varepsilon$

$$
J(W, \varepsilon, \phi)=2\left(\rho^{\prime}\right)^{2}-\rho^{\prime \prime} \rho+\rho^{2}>0
$$

for every $W$ and $\phi$; see [Ga2, p. 25].

Computing the derivatives,

$$
\begin{aligned}
\rho^{\prime}(\phi) & =-\frac{d}{d \phi}\left(\rho_{W}(\phi)\right), \\
\rho^{\prime \prime}(\phi) & =-\frac{d^{2}}{d^{2} \phi}\left(\rho_{W}(\phi)\right) .
\end{aligned}
$$

To estimate $\rho^{\prime}$ and $\rho^{\prime \prime}$, consider

$$
\begin{aligned}
\left|\frac{d}{d \phi}\left(\rho_{W}(\phi)\right)\right| & =\left|\sum_{i=1}^{n} \frac{\partial}{\partial x_{i}}\left(f_{\varepsilon}(\theta) r^{-n+1}\right)^{\wedge}(x)\right|\left|\frac{d x_{i}}{d \phi}\right| \\
& \leq 2\left|\sum_{i=1}^{n} \frac{\partial}{\partial x_{i}}\left(f_{\varepsilon}(\theta) r^{-n+1}\right)^{\wedge}(x)\right|,
\end{aligned}
$$

since $x=\xi_{1} \cos \phi+\xi_{2} \sin \phi$ and

$$
\left|\frac{d x_{i}}{d \phi}\right|=\left|-\xi_{1, i} \sin \phi+\xi_{2, i} \cos \phi\right| \leq 2
$$

Similarly,

$$
\left|\frac{d^{2}}{d \phi^{2}}\left(\rho_{W}(\phi)\right)\right| \leq 4\left|\sum_{i, j=1}^{n} \frac{\partial^{2}}{\partial x_{i} \partial x_{j}}\left(f_{\varepsilon}(\theta) r^{-n+1}\right)^{\wedge}(x)\right| .
$$

By Lemma 2.4 we have $\rho^{\prime}=O\left(|\ln \varepsilon| \varepsilon^{n-3}\right)$ and $\rho^{\prime \prime}=O\left(\varepsilon^{n-4}\right)$. Since these estimates are uniform with respect to $\phi$ and $W$, it follows that, for small enough $\varepsilon$, $J(W, \varepsilon, \phi)>0$ for every $\phi$ and $W$. 
In [K1, Lemma 1] the following was proved. Let $f$ be an even continuous homogeneous function of degree $-n+1$ on $\mathbb{R}^{n} \backslash\{0\}$. Then $\hat{f}$ is a continuous function outside of the origin and for every $\xi \in S^{n-1}$,

$$
\hat{f}(\xi)=\pi \int_{S^{n-1} \cap\{(\theta, \xi)=0\}} f(\theta) d \theta .
$$

Using this formula,

$$
\left(\left(1-f_{\varepsilon}(\theta)\right) r^{-n+1}\right)^{\wedge}(x)=\pi \int_{S^{n-1} \cap\{(x, \theta)=0\}}\left(1-f_{\varepsilon}(\theta)\right) d \theta .
$$

Lemma 2.6. For every $(n-1)$-dimensional subspace $V$ of $\mathbb{R}^{n}$, the body $K \cap V$ is an intersection body.

Proof. Fix an $(n-1)$-dimensional subspace $V$ of $\mathbb{R}^{n}$. By the definition (1) of the body $K$ and formula (6)

$$
\frac{1}{\pi}\|x\|_{K}^{-1}=\int_{S^{n-1} \cap\{(x, \theta)=0\}}\left(1-f_{\varepsilon}(\theta)\right) d \theta .
$$

In particular, for $x \in V$,

$$
\frac{1}{\pi}\|x\|_{K \cap V}^{-1}=\int_{S^{n-1} \cap\{(x, \theta)=0, x \in V\}}\left(1-f_{\varepsilon}(\theta)\right) d \theta,
$$

where $\{(x, \theta)=0, x \in V\}$ is the hyperplane of all $\theta$ that are perpendicular to $x$ for a fixed $x \in V$.

Let us change coordinates from $\theta \in S^{n-2}=S^{n-1} \cap\{(x, \theta)=0, x \in V\}$ to $\phi \in[0, \pi]$ and $\xi \in S^{n-3}=S^{n-2} \cap\{(x, \theta)=0, x \in V\}$. The Jacobian is equal to $(\sin \phi)^{n-3}$; cf. [M, p. 1]. So

$$
\begin{aligned}
& \frac{1}{\pi}\|x\|_{K \cap V}^{-1}=\int_{S^{n-2} \cap\{(x, \theta)=0, x \in V\}}\left(\int_{0}^{\pi}\left(1-f_{\varepsilon}(\xi, \phi)\right)(\sin \phi)^{n-3} d \phi\right) d \xi \\
= & \int_{S^{n-2} \cap\{(x, \theta)=0, x \in V\}}\left(\int_{0}^{\pi}(\sin \phi)^{n-3} d \phi-\int_{0}^{\pi} f_{\varepsilon}(\xi, \phi)(\sin \phi)^{n-3} d \phi\right) d \xi .
\end{aligned}
$$

Taking the Fourier transform of both sides, as functions of the variable $x \in V$, and using (5)

$$
\left(\|x\|_{K \cap V}^{-1}\right)^{\wedge}(\theta)=\pi \int_{0}^{\pi}(\sin \phi)^{n-3} d \phi-\pi \int_{0}^{\pi} f_{\varepsilon}(\theta)(\sin \phi)^{n-3} d \phi
$$

for $\theta \in S^{n-2}$.

By the definition, $f_{\varepsilon}$ is non-zero only in an $\varepsilon$-neighborhood of $x_{0}$, so there exists a set $R_{\varepsilon} \subset[0, \pi]$ such that $f_{\varepsilon}(\xi, \phi)=0$ for $\phi \in[0, \pi] \backslash R_{\varepsilon}$. Also, $\left|f_{\varepsilon}\right| \leq 2$ and $\left|R_{\varepsilon}\right|$, the length of the one-dimensional set $R_{\varepsilon}$, is of the order $\varepsilon$. Therefore,

$$
\begin{aligned}
\int_{0}^{\pi} f_{\varepsilon}(\xi, \phi)(\sin \phi)^{n-3} d \phi & =\int_{R_{\varepsilon}} f_{\varepsilon}(\xi, \phi)(\sin \phi)^{n-3} d \phi \\
& \leq 2\left|R_{\varepsilon}\right|=C \varepsilon,
\end{aligned}
$$

where $C$ does not depend of the choice of $V$.

Since $\int_{0}^{\pi}(\sin \phi)^{n-3} d \phi$ is equal to some positive constant depending on $n$ only,

$$
\int_{0}^{\pi}(\sin \phi)^{n-3} d \phi-\int_{0}^{\pi} f_{\varepsilon}(\theta)(\sin \phi)^{n-3} d \phi>0
$$


for a sufficiently small $\varepsilon$, which means we can find $\varepsilon$ small enough that $\left(\|x\|_{K \cap V}^{-1}\right)^{\wedge}(\theta)$ $>0$ for all $\theta$. Therefore by [K2, Thm. 1], for small enough $\varepsilon$, for every $(n-1)$ dimensional subspace $V$ of $\mathbb{R}^{n}$, the body $K \cap V$ is an intersection body.

Now Theorem 2.1 follows from Lemmas 2.3, 2.5 and 2.6.

Remark 1. By a result of Neyman [N], for any finite system of equations and inequalities involving the norms, there exists a subspace of $L_{p}, 0<p<2$, that does not satisfy this system. Since, by $\mathrm{K} 2$, the unit ball of every finite-dimensional subspace of $L_{p}, 0<p<2$, is an intersection body, we conclude that the class of intersection bodies cannot be characterized by a finite number of equations or inequalities.

Remark 2. For $n=5$ the fact that there exists a non-intersection body whose central sections are intersection bodies follows from the solution of the Busemann-Petty problem, since every four-dimensional symmetric convex body is an intersection body; see GKS], Zh2].

Remark 3. It is known that the polar of zonoid is an intersection body (see $\mathrm{K5}$, p. 127]), but the converse is not true, and the polar of an intersection body may not be a zonoid, as for example a cube in $\mathbb{R}^{4}$. Therefore, the result in this paper does not follow the result of W.Weil on zonoids by applying duality argument, and a new example should be constructed.

\section{ACKNOWLEDGMENTS}

The author is thankful to A.Koldobsky and V.Yaskin for their valuable help in preparation of this paper. The author is grateful to the referee for many suggestions that helped to improve this paper. Research was supported in part by the European Network PHD, FP6 Marie Curie Actions, RTN, Contract MCRN - 511953.

\section{REFERENCES}

[B] M. BURGER, Finite sets of piecewise linear inequalities do not characterize zonoids, Arch. Math. (Basel) 70 (1998), no. 2, 160-168. MR.1491464 (99b:52007)

[BP] H. Busemann, C. M. Petty, Problems on convex bodies, Math. Scand. 4 (1956), 88-94. MR0084791 (18:922b)

[Ga1] R. J. GARDNeR, Intersection bodies and the Busemann-Petty problem, Trans. A.M.S. 342 (1994), 435-445. MR1201126 (94e:52008)

[Ga2] R. J. Gardner, Geometric tomography, Cambridge University Press, 1995. MR 1356221 (96j:52006)

[GKS] R. J. Gardner, A. Koldobsky, T. Schlumprecht, An analytic solution to the Busemann-Petty problem on sections of convex bodies, Annals of Math. 149 (1999), 691703. MR:1689343 (2001b:52011)

[Gr] H. Groemer, Geometric application of Fourier series and shperical harmonics, Cambridge University Press, New York, 1996. MR1412143 (97j:52001)

[GS] I. M. Gelfand And G. E. Shilov, Generalized functions, vol.1 Properties and Operations, Academic Press, New York and London, 1964. MR0166596 (29:3869)

[GV] I. M. Gelfand, N. Ya. Vilenkin, Generalized functions, vol.4. Applications of harmonic analysis, Academic Press, New York, 1964. MR0173945 (30:4152)

[K1] A. Koldobsky, An application of the Fourier transform to sections of star bodies, Israel J. Math. 106 (1998), 157-164. MR1656857 (99k:42011)

[K2] A. Koldobsky, Intersection bodies, positive definite distributions and the Busemann-Petty problem, Amer. J. Math. 120 (1998), 827-840. MR.1637955(99i:52005)

[K3] A. Koldobsky, Second derivative test for intersection bodies, Adv. Math. 136 (1998), 15-25. MR 1623670 (99j:52014) 
[K4] A. Koldobsky, Positive definite distributions and subspaces of $L_{-p}$ with applications to stable processes, Canad. Math. Bull. 42 (3) (1999), 344-353. MR.1703694(2001i:42014)

[K5] A. Koldobsky, Fourier analysis in convex geometry, Amer. Math. Society, Providence, RI, 2005. MR2132704 (2006a:42007)

[Lu] E. Lutwak, Intersection bodies and dual mixed volumes, Advances in Math. 71 (1988), 232-261. MR0963487 (90a:52023)

[M] C. Müller, Spherical harmonics, Springer-Verlag, Berlin, Heidelberg, New York, 1966. MR0199449 (33:7593)

[N] A. Neyman, Representation of $L_{p}$-norms and isometric embedding in $L_{p}$-spaces, Israel Journal of Math. 48 Nos. 2-3 (1984), 129-138. MR0770695 (86g:46033)

[W] W. WeIL, Zonoide und verwandte Klassen konvexer Körper, Monatsh. Math. 94 (1982), 73-84. MR.0670016 (84e:52008)

[Zh1] Gaoyong Zhang, Intersection bodies and Busemann-Petty inequalities in $\mathbb{R}^{n}$, Annals of Math. 140 (1994), 331-346. MR1298716 (95i:52004)

[Zh2] Gaoyong Zhang, A positive answer to the Busemann-Petty problem in $\mathbb{R}^{n}$, Annals of Math. 149 (1999), 535-543. MR.1689339 (2001b:52010)

Department of Mathematics, University of Missouri, Columbia, Missouri 65211

E-mail address: yaskinam@math.missouri.edu

Current address: Department of Mathematics, University of Oklahoma, Norman, Oklahoma 73019

E-mail address: myaskina@math.ou.edu 